\title{
Tolvaptan Prolongs Blockage of the Vasopressin Type II Receptor Over 24 Hours in Responders With Stage D Heart Failure
}

\author{
Teruhiko Imamura, ${ }^{1} \mathrm{MD}$, Koichiro Kinugawa, ${ }^{1} \mathrm{MD}$, and Issei Komuro, ${ }^{2} \mathrm{MD}$
}

\begin{abstract}
SUMMARY
The urine aquaporin-2 (U-AQP2) level relative to the plasma arginine vasopressin (P-AVP) level is a novel predictor of the responsiveness to the vasopressin type 2 receptor (V2R) antagonist tolvaptan (TLV). However, little has been reported about the concentration-time profile of U-AQP2 after TLV treatment. We evaluated 24 patients with decompensated stage D heart failure (HF) who had received $3.75 \mathrm{mg} /$ day of TLV on a de novo basis for $>7$ days to treat congestion refractory to conventional diuretics. Seventeen patients were TLV-responders, whose 24-hour urine volume (UV) increased after TLV initiation; the other 7 patients were TLV-non-responders. The U-AQP2 of the TLV-responders, corrected for the urine creatinine concentration, decreased significantly at 4 hours after TLV administration without returning to the day-1 morning level on the morning of day-7. The TLV-non-responder U-AQP2 levels remained low even before the TLV treatment. On the morning of day-7, the TLV-responder U-AQP2/P-AVP ratio was comparable to that of the TLV-non-responders. Among 18 patients (11 responders and 7 non-responders), the day-7 TLV trough concentration was $64 \pm 62 \mathrm{ng} / \mathrm{mL}$ and was negatively correlated with the estimated glomerular filtration rate (eGFR). TLV has antagonistic effects on the V2R over 24 hours in TLV-responders with advanced heart failure and chronic kidney disease, probably due to persistently elevated blood TLV concentration. The unresponsiveness to TLV in the TLV-non-responders is not attributable to malabsorption. (Int Heart J 2016; 57: 41-46)
\end{abstract}

Key words: Congestion, Drug responder, Renal failure

$\mathrm{T}$ he arginine vasopressin (AVP) type 2 antagonist tolvaptan (TLV) is known to ameliorate congestion and to normalize hyponatremia, thereby preserving hemodynamics and renal function in patients with congestive heart failure (HF) refractory to a considerable number of conventional diuretics. ${ }^{1-10)}$ In contrast, we sometimes experience TLV-non-responders in our daily clinical practice. ${ }^{11)}$

We previously proposed a novel predictor of responsiveness to TLV, ie, the baseline urine aquaporin-2 (U-AQP2) level relative to the plasma AVP (P-AVP) level, which may indicate a preserved vasopressin type 2 receptor (V2R)-related signaling pathway in the principal cells of the renal collecting duct. ${ }^{12,13)}$ However, little is known about the concentration-time profile of U-AQP2 after the initiation of TLV treatment. Although Martin, et al observed the changes of U-AQP2 after the initiation of V2R antagonist, the responsiveness to TLV was not discussed. ${ }^{14)}$ Therefore, in this study, we investigated the $\mathrm{U}-\mathrm{AQP} 2$ profile and the relationship between U-AQP2 and other clinical parameters after the initiation of TLV treatment in TLV-responders and TLV-non-responders.

\begin{abstract}
Methods
Patient selection: Among the patients who had been hospitalized at our institute between August 2013 and August 2014 for decompensated HF, data were obtained from 24 patients who had received $3.75 \mathrm{mg} /$ day of TLV on a de novo basis for $>7$ days. All patients presented New York Heart Association class III/IV symptoms accompanied by symptomatic congestion such as lower limb edema, pulmonary congestion, or jugular venous distention. The patients were refractory to guideline-directed medical treatment with a considerable number of conventional diuretics, including loop diuretics and/or thiazides in addition to the appropriate restriction of sodium and water intake. Therefore, all patients were classified as suffering from stage D heart failure ${ }^{15)}$ Patients with any of the following conditions were excluded from the study and did not receive TLV: severe systemic infection or inflammation, severe stenotic valvular disease, end-stage renal failure or on hemodialysis, acute coronary syndrome occurring within a month, the need for the use of mechanical support, hypovolemia, impaired consciousness with lack of thirst, or hypernatremia (> $145 \mathrm{mEq} / \mathrm{L}$ of serum sodium) as previously described. ${ }^{11)}$ The present study complies with the Declaration of Helsinki and the institutional review board of University of Tokyo approved the research
\end{abstract}

From the Departments of ${ }^{1}$ Therapeutic Strategy for Heart Failure and ${ }^{2}$ Cardiovascular Medicine, Graduate School of Medicine, The University of Tokyo, Tokyo, Japan.

Address for correspondence: Koichiro Kinugawa, MD, Department of Therapeutic Strategy for Heart Failure, Graduate School of Medicine, The University of Tokyo,

7-3-1 Hongo, Bunkyo-ku, Tokyo 113-8655, Japan. E-mail: kinugawa-tky@umin.ac.jp

Received for publication July 27, 2015. Revised and accepted August 9, 2015.

Released in advance online on J-STAGE January 8, 2016.

All rights reserved by the International Heart Journal Association. 
protocol. Informed consent was obtained from all patients prior to enrollment.

Variables evaluated: Baseline demographic data were obtained within 24 hours prior to the TLV treatment. Blood samples, including those for the P-AVP measurement, were obtained in the early morning before the ingestion of medication (including diuretics) and after a 15-minute rest in the supine position. ${ }^{16)}$ Urine samples were collected at 3 time points: 1) in the early morning before the administration of any medication, including TLV and other diuretics (day-1 morning); 2) at 4 hours following the first TLV administration (day-1 4 hours); and 3 ) in the early morning at day-7 before the administration of any drugs, including TLV (day-7 morning). The U-AQP2 level was measured using a sandwich enzyme-linked immunosorbent assay (human aquaporin-2 ELISA kit, LSI Medience) and was corrected for the urine creatinine (U-Cre) concentration to allow for a quantitative comparison as previously described. ${ }^{12,17)}$ The urine volume (UV) collected during 24 hours after the initiation of the TLV treatment (day-1) was compared with that of the previous 24 hours (day-0). The patients, whose 24-hour UV at day-1 increased compared with that at day-0, were defined as TLV-responders (UV-defined responders). The other patients were assigned to the TLV-non-responders group (UV-defined non-responders). ${ }^{11)}$ The TLV trough concentration was measured in the blood samples obtained from 18 patients at day-7 in the morning using liquid chromatography-tandem mass spectrometry. ${ }^{18)}$

Statistical analyses: The data are expressed as the mean $\pm \mathrm{SD}$, unless otherwise indicated. The continuous variables were compared using unpaired Student's $t$-test or Mann-Whitney $U$ test as appropriate. The categorical variables were compared using the $X^{2}$ or Fisher's exact test as appropriate. Dunnett's test was used to compare the continuous variables among the several time points measured if repeated analysis of variance showed significance. Pearson's correlation coefficient was used to assess the correlation between 2 groups. All statistical analyses were performed using SPSS statistics software (version 22; SPSS, Inc., Chicago, IL, USA). All statistical tests were 2 -tailed and $P<0.05$ was considered significant.

\section{Results}

Baseline characteristics: The baseline variables before the initiation of the TLV therapy are presented in Table I. The mean population age was $54 \pm 20$ years and the average estimated glomerular filtration ratio (eGFR) was $52 \pm 15 \mathrm{~mL} / \mathrm{kg} / 1.73 \mathrm{~m}^{2}$ (range: $23.8-69.4 \mathrm{~mL} / \mathrm{kg} / 1.73 \mathrm{~m}^{2}$ ). The TLV-responders had a

Table I. Baseline Variables Before the Initiation of TLV

\begin{tabular}{|c|c|c|c|c|}
\hline & $\begin{array}{c}\text { Total } \\
(n=24)\end{array}$ & $\begin{array}{l}\text { Responders } \\
\quad(n=17)\end{array}$ & $\begin{array}{l}\text { Non-responders } \\
\qquad(n=7)\end{array}$ & $P$ \\
\hline \multicolumn{5}{|l|}{ Demographics } \\
\hline Age, years & $54 \pm 20$ & $51 \pm 17$ & $62 \pm 24$ & 0.202 \\
\hline Male, $n(\%)$ & $19(79)$ & $13(76)$ & $6(86)$ & 0.612 \\
\hline BMI & $22.9 \pm 3.4$ & $23.4 \pm 3.2$ & $22.8 \pm 2.9$ & 0.215 \\
\hline $\mathrm{sBP}, \mathrm{mmHg}$ & $96 \pm 13$ & $95 \pm 13$ & $100 \pm 14$ & 0.441 \\
\hline HR, bpm & $82 \pm 12$ & $82 \pm 12$ & $83 \pm 11$ & 0.799 \\
\hline \multicolumn{5}{|l|}{ Medications } \\
\hline $\mathrm{BB}, n(\%)$ & $21(88)$ & $15(88)$ & $6(86)$ & 0.865 \\
\hline ACEI, $n(\%)$ & $16(67)$ & $11(65)$ & $5(71)$ & 0.751 \\
\hline $\mathrm{AA}, n(\%)$ & $15(63)$ & $12(71)$ & $3(43)$ & 0.202 \\
\hline Furosemide, $n(\%)$ & $24(100)$ & $17(100)$ & $7(100)$ & 1.000 \\
\hline Furosemide, mg/day & $65 \pm 20$ & $67 \pm 21$ & $59 \pm 17$ & 0.356 \\
\hline \multicolumn{5}{|l|}{ Laboratory } \\
\hline Hemoglobin, g/dL & $11.2 \pm 2.1$ & $11.6 \pm 2.4$ & $10.3 \pm 0.9$ & 0.087 \\
\hline Serum albumin, g/dL & $3.4 \pm 0.6$ & $3.4 \pm 0.5$ & $3.3 \pm 0.7$ & 0.630 \\
\hline Serum sodium, $\mathrm{mEq} / \mathrm{L}$ & $134 \pm 6$ & $134 \pm 7$ & $135 \pm 4$ & 0.757 \\
\hline $\mathrm{eGFR}, \mathrm{mL} / \mathrm{kg} / 1.73 \mathrm{~m}^{2}$ & $52 \pm 15$ & $61.4 \pm 45.1$ & $35.5 \pm 11.1$ & $0.001^{*}$ \\
\hline Serum TB, mg/dL & $1.3 \pm 0.9$ & $1.4 \pm 0.9$ & $1.3 \pm 0.8$ & 0.856 \\
\hline Plasma BNP, pg/mL & $672 \pm 648$ & $711 \pm 658$ & $579 \pm 663$ & 0.659 \\
\hline Plasma AVP, ng/mL & $3.9 \pm 1.4$ & $4.1 \pm 1.3$ & $3.5 \pm 1.8$ & 0.383 \\
\hline \multicolumn{5}{|l|}{ Urine variables } \\
\hline U-OSM, mOsm/L & $417 \pm 122$ & $464 \pm 105$ & $302 \pm 79$ & $0.001^{*}$ \\
\hline U-AQP2/U-Cre, $\times 10^{2}$ & $60 \pm 42$ & $76 \pm 40$ & $22 \pm 9$ & $<0.001^{*}$ \\
\hline U-AQP2/P-AVP, $\times 10^{3}$ & $1.1 \pm 0.8$ & $1.5 \pm 0.7$ & $0.3 \pm 0.1$ & $<0.001^{*}$ \\
\hline \multicolumn{5}{|l|}{ Echocardiography } \\
\hline LVDd, mm & $60 \pm 14$ & $61 \pm 15$ & $57 \pm 15$ & 0.317 \\
\hline LVEF, \% & $35 \pm 18$ & $34 \pm 18$ & $37 \pm 19$ & 0.234 \\
\hline \multicolumn{5}{|l|}{ NYHA class } \\
\hline Class III, $n(\%)$ & $11(46)$ & $7(41)$ & $4(86)$ & 0.476 \\
\hline Class IV, $n(\%)$ & $13(54)$ & $10(59)$ & $3(43)$ & 0.476 \\
\hline
\end{tabular}

$\mathrm{BMI}$ indicates body mass index; $\mathrm{sBP}$, systolic blood pressure; $\mathrm{HR}$, heart rate; $\mathrm{BB}, \beta$-blocker; ACEI, angiotensin converting enzyme inhibitor; AA, aldosterone antagonist; eGFR, estimated glomerular filtration rate; TB, total bilirubin; BNP, B-type natriuretic peptide; AVP, arginine vasopressin; U-OSM, urine osmolality; U-AQP2, urine aquaporin-2; U-Cre, urine creatinine; LVDd, left ventricular diastolic diameter; LVEF, left ventricular ejection fraction; NYHA, New York Heart Association. ${ }^{*} P<0.05$ by the unpaired $t$-test. 

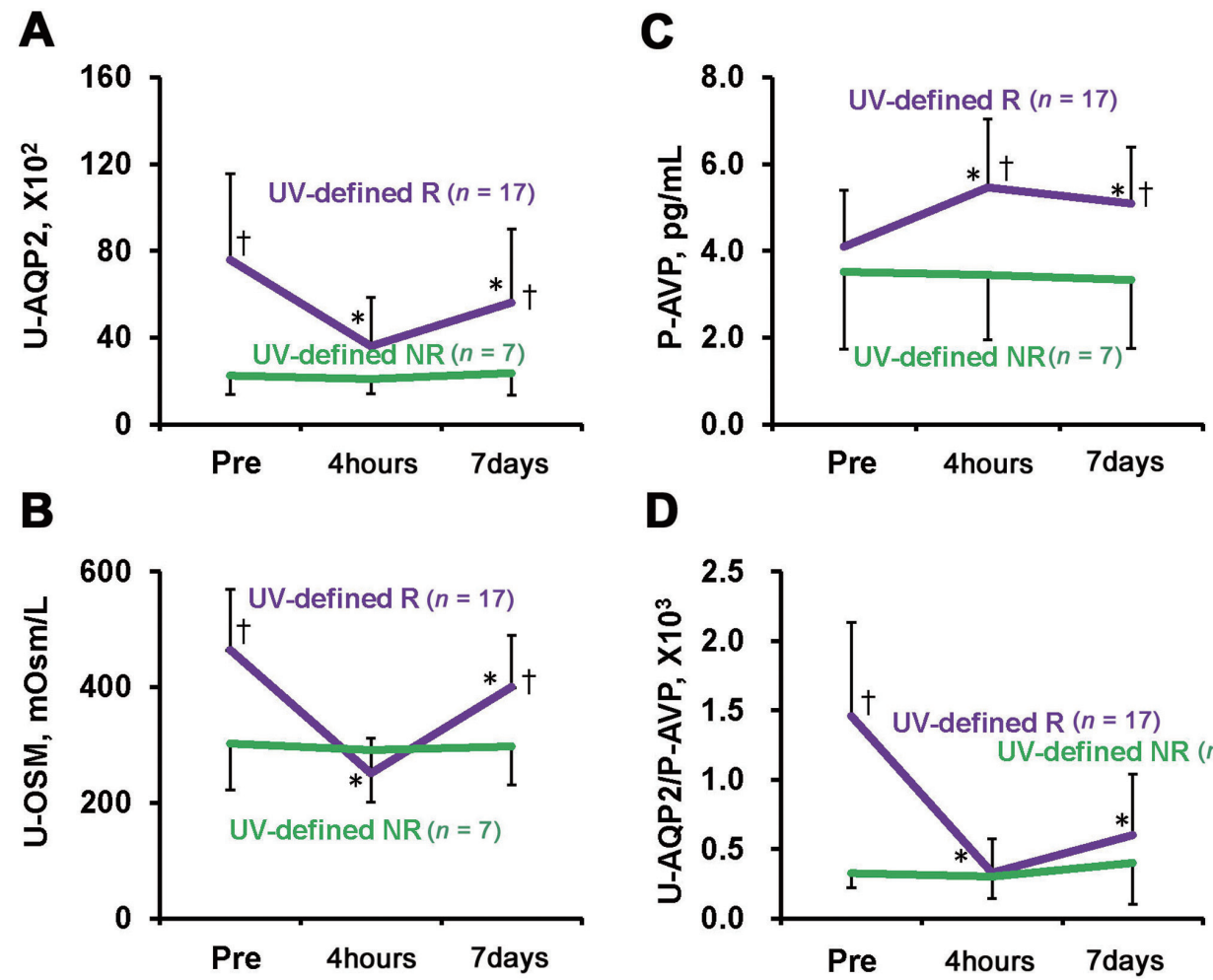

D

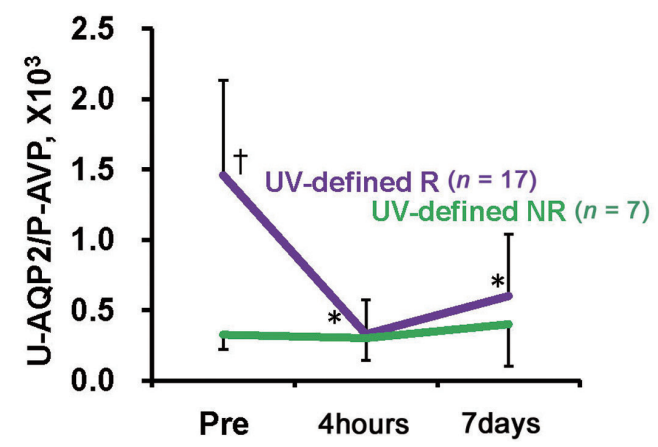

Figure 1. The concentration-time profiles of U-AQP2 (A), U-OSM (B), P-AVP (C), and U-AQP/P-AVP (D) during TLV therapy in the TLV-responders and TLV-non-responders. R indicates TLV-responder; and NR, TLV-non-responder. ${ }^{*} P<0.05$ compared with the baseline variable as determined by Dunnett' test if repeated analysis of variance showed significance. ${ }^{\dagger} P$ $<0.05$ compared with the TLV-non-responders as determined by Tukey's test if 2-way repeated analysis of variance showed significance.

significantly higher urine osmolality (U-OSM) level, U-AQP2 level corrected for U-Cre, and U-AQP2/P-AVP ratio than those of the TLV-non-responders ( $P<0.05$ for all comparisons).

Time-dependency of U-AQP2 levels and of other variables: In the TLV-responders, both the U-AQP2 level (Figure 1A) and the U-OSM level (Figure 1B) markedly decreased at 4 hours following the first administration of TLV $(P<0.05$ for both analyses). The U-AQP2 and U-OSM levels at day-7 before the administration of TLV were significantly lower as compared with the day-1 morning levels ( $P<0.05$ for both analyses). In contrast, in the TLV-non-responders, the U-AQP2 and U-OSM levels remained low and were not affected by the TLV treatment at all 3 time points measured. The P-AVP level was increased at 4 hours after the first administration of TLV and remained at a higher level at day-7 in the TLV-responders (Figure $1 \mathrm{C})$. As a result, the TLV-responder U-AQP2/P-AVP ratio was similar to that of the TLV-non-responders at day-7 morning (Figure 1D; $0.6 \pm 0.4$ versus $0.4 \pm 0.3 \times 10^{3}, P=0.087$ ).

TLV trough concentration at day-7: The TLV trough concentration was measured in 18 patients, including 11 responders and 7 non-responders, at day-7 morning $(64 \pm 62 \mathrm{ng} / \mathrm{mL}$ on average). No significant differences were observed in the TLV trough concentration between the TLV-responders and TLVnon-responders ( $43 \pm 38$ versus $98 \pm 80 \mathrm{ng} / \mathrm{mL}, P=0.182)$. As shown in Table II, only the eGFR was significantly correlated with the TLV trough levels at day-7 morning among both responders and non-responders to TLV (Figure 2; $P=0.019, r=$
Table II. Relationship Between Baseline Variables and Trough Level of TLV at 1 Week Following TLV Administration

\begin{tabular}{llr}
\hline & $P$ & $r$ \\
\hline Demographics & & \\
$\quad$ Age, years & 0.211 & -0.312 \\
BMI & 0.132 & 0.371 \\
sBP, mmHg & 0.763 & -0.076 \\
$\quad$ HR, bpm & 0.215 & -0.307 \\
Medications & & \\
$\quad$ Furosemide, mg/day & 0.143 & -0.365 \\
Laboratory & & \\
$\quad$ Hemoglobin, g/dL & 0.525 & -0.162 \\
$\quad$ Serum albumin, g/dL & 0.2021 & 0.316 \\
$\quad$ Serum sodium, mEq/L & 0.734 & -0.086 \\
$\quad$ eGFR, mL/kg/1.73 m ${ }^{2}$ & $0.019^{*}$ & -0.545 \\
$\quad$ Serum total bilirubin, mg/dL & 0.658 & 0.112 \\
$\quad$ Plasma BNP, pg/mL & 0.611 & -0.129 \\
$\quad$ Plasma AVP, ng/mL & 0.434 & 0.243 \\
Echocardiography & & \\
$\quad$ LVDd, mm & 0.243 & 0.143 \\
$\quad$ LVEF, \% & 0.265 & 0.176 \\
\hline A &
\end{tabular}

Abbreviations as in Table I. " $P<0.05$ by Pearson's correlation coefficient.

-0.545). The TLV trough level was negatively correlated with U-AQP2 in the responders $(P=0.042, r=-0.342)$, but not in the non-responders $(P=0.946, r=0.065)$. 


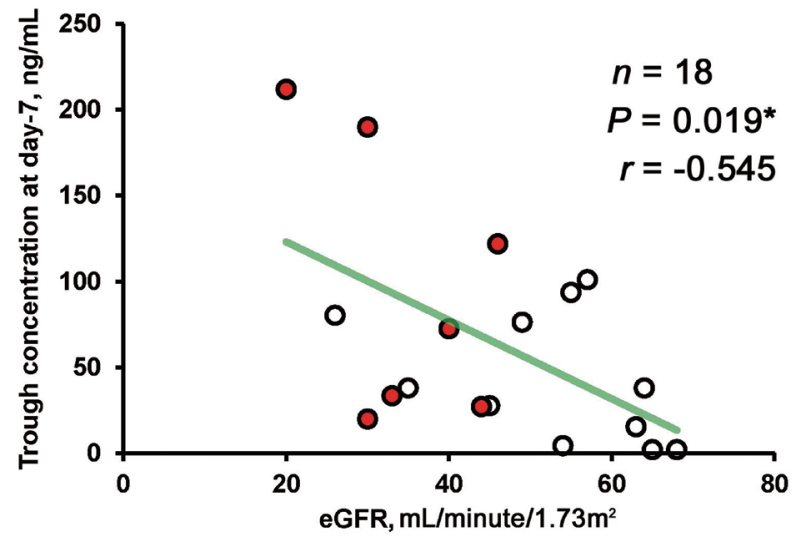

Figure 2. The relationship between the eGFR and the TLV trough concentration at day-7. White and red circles indicate the TLV-responders and TLV-non-responders, respectively. ${ }^{*} P<0.05$ as determined by Pearson's correlation coefficient.

\section{Discussion}

This study showed that the U-AQP2 level decreased 4 hours after the administration of TLV in the TLV-responders, whereas it remained low in the TLV-non-responders. Furthermore, the responder U-AQP2 level at day-7 did not fully return to the baseline level. The TLV trough concentration at day-7 was measured in 18 patients: that of the TLV-non-responders was as high as that of the TLV-responders.

Excretion of AQP2 in urine: The precise function of AQP2 has been investigated in several early, classical analysis studies. AQP2 is the AVP-regulated water channel that mediates water transport across the apical plasma membrane of the renal collecting duct. ${ }^{19)}$ The number of AQP-2 water channels determines the water permeability of the apical membrane and is principally regulated by the P-AVP level. ${ }^{20)}$ AVP regulates the translocation of AQP-2 proteins from cytosolic vesicles to the apical plasma membranes, a shuttle trafficking process that regresses with the suppression of AVP stimulation. ${ }^{21)}$ Approximately $3 \%$ of AQP2 in the kidney is excreted in the urine daily; this fraction is not affected by the various conditions present in patients. ${ }^{22)}$ Therefore, U-AQP2 is considered a marker of collecting duct responsiveness to AVP. ${ }^{12,23)}$

U-AQP2 and responsiveness to TLV: We previously demonstrated that responsiveness to TLV is largely dependent on the viability of the collecting duct, ${ }^{11)}$ and that TLV-responders can be discriminated from TLV-non-responders by measuring their respective baseline $\mathrm{U}-\mathrm{AQP} 2$ relative to $\mathrm{P}-\mathrm{AVP}$ level prior to TLV administration. ${ }^{12)}$ However, few reports exist on the concentration-time profile of U-AQP2 after the initiation of TLV therapy. Martin, et al previously reported that the U-AQP2 level decreased significantly after the administration of TLV, ${ }^{14}$ however, the potential relationship between the changes in the $\mathrm{U}-\mathrm{AQP} 2$ level and the responsiveness to TLV remains unknown.

Acute U-AQP2 response: In the TLV-responders, we observed decreases in the U-AQP level at 4 hours following the administration of TLV. The 4-hour time point was chosen based on previous studies showing that both the maximum plasma concentration of TLV and the maximum UV response occurred at 4 hours. ${ }^{24)}$ AVP stimulates the synthesis of AQP2 and a part of this is excreted in urine. A 4-hour observation time is enough to see the changes in transcription of AQP2. We confirmed that the inhibition of the V2R by TLV resulted in a marked decrease in the U-AQP2 level, suggesting a reduced activation, ie, less phosphorylation and translocation, of AQP2. Importantly, the altered concentration of AQP2 in the urine was not merely a result of aquaresis but rather a consequence of a real decrease in solute excretion, since we corrected the U-AQP2 concentration for that of urine creatinine. In contrast, an already low U-AQP2 level was observed in the TLV-non-responders before the TLV treatment, which was not decreased after TLV administration. This finding suggests that V2R-mediated signaling pathways are not active in the TLV-non-responders, or at least do not propagate through the principal cells of the renal collecting duct. We speculate that the unresponsiveness to TLV is attributable to a certain dysfunction of the collecting duct, which is often observed in aged patients with decompensated $\mathrm{HF}^{11)}$

Mid-term U-AQP2 response: The P-AVP level increased at day-7. Although the precise mechanism underlying the increase in P-AVP after the administration of TLV remains unknown, such an increase is consistently observed. ${ }^{8)}$ Although TLV therapy elevates serum osmolality by increasing serum sodium levels, it is usually insufficient to stimulate the excretion of AVP. ${ }^{16)}$ P-AVP can also be secreted by non-osmotic stimulation, including hypotension, but TLV rarely induces hemodynamic compromise even in patients with advanced $\mathrm{HF}^{5 \text { ) }}$

Despite the increase in the P-AVP level at day-7, the UAQP2 level did not return to the baseline level after 7 days of TLV administration (75\% compared with that at baseline) in the TLV-responders, indicating that the AQP2 excretion in urine was considerably suppressed after 24 hours of TLV administration. Since the antagonistic effect of TLV is transient (personal communication from Otsuka Pharmaceuticals), we hypothesize that TLV at its measured trough concentration at day-7 can inhibit the V2R in the presence of AVP. Here, we measured the TLV trough concentration at day-7 because the plasma TLV concentration is known to remain stable for at least 7 days. ${ }^{18)}$

At day-7 morning, the average TLV trough concentration in 18 patients, including 11 responders and 7 non-responders, was $64 \mathrm{ng} / \mathrm{mL}$, which was higher than those of healthy subjects or HF patients with normal kidney function. ${ }^{8,25)}$ Van Wart, et al observed a more elevated peak plasma concentration of TLV in HF patients with or without liver dysfunction compared with that of healthy subjects. ${ }^{26)}$ Moreover, Kida, et al reported an elevated TLV trough level at day-7 in HF patients with chronic kidney disease. ${ }^{18)}$ Most of the patients in the current study had chronic kidney disease in addition to stage D HF. Although the precise mechanism underlying the persistently elevated TLV trough concentration in such conditions is still unknown, a decreased systemic circulation and over volume due to renal impairment may contribute to the reduced metabolism of TLV and elevated blood TLV concentration. The TLV trough level in the TLV-non-responders was similar to that of the TLV-responders. This result supports our hypothesis that the unresponsiveness to TLV was not attributable to the low blood concentration of TLV due to malabsorption, but rather to the dysfunction of the renal collecting duct. ${ }^{11,12)}$ Accordingly, the up-titration of TLV may not be effective in the TLV-non-responders. 
We previously proposed the criterion to predict responsiveness to TLV: the U-AQP2 level relative to the P-AVP level, which can be obtained before the initiation of TLV therapy. ${ }^{12)}$ As shown in the present study, the U-AQP2/P-AVP ratio at day-7 was similarly decreased and low in the TLV-responders and the TLV-non-responders. It is therefore important that these biomarkers should be measured before the initiation of TLV treatment.

Study limitations: 1) The present study was performed in a small population in a single study center. Hence, the findings should be confirmed in a future multi-center, large-scale population study. 2) The present study was conducted at a fixed TLV dose. Dose comparison analyses may provide additional information. However, the adjustment of each different TLV dose to the patients' background may be difficult in a real clinical setting. 3) We analyzed the mid-term U-AQP2 response at 7 days after the initiation of the TLV treatment, assuming that the TLV trough concentration had reached steady state at that time point. Determining the U-AQP2 levels after long-term V2R inhibition by TLV (eg, over years) is the next step to be taken. 4) We enrolled patients with New York Heart Association class III or IV and who had comorbid reduced renal function. Therefore, our results cannot be extrapolated to relatively less sick patients with a normal eGFR. 5) Martin, et al observed the changes of U-AQP2 every 2 hours until 8 hours after V2R antagonist administration, ${ }^{14)}$ whereas we measured it at 4 hours and day-7. AVP can stimulate synthesis of AQP2, and 4 hours may be enough to observe increased urinary secretion of AQP2 by way of AVP-enhanced transcription of AQP2. The continuous measurement of UV using a urethral catheter may provide information regarding the precise responsiveness to TLV, although the use of a dwelling urethral catheter has become uncommon. 6) U-OSM data are obtained more rapidly than U-AQP2. U-OSM may have an advantage in the rapid prediction of responsiveness to TLV. However, U-AQP2 relative to P-AVP had better predictability compared with U-OSM or U-OSM/P-AVP (area under curve for predictability, 1.000, 0.871, and 0.639, respectively). 7) Baseline U-AQP2/P-AVP was obtained in the responders receiving anti-HF treatment including diuretics $\left(n=17,1.5 \pm 0.7 \times 10^{3}\right)$, and the data represent chronic effects of baseline anti-HF treatment on U-AQP2/ $\mathrm{P}-\mathrm{AVP}$. We also obtained a comparable level of U-AQP2/PAVP in a healthy population receiving no anti-HF treatment $(n$ $=5,2.4 \pm 1.4 \times 10^{3}, P=0.635$ ). Therefore, general anti-HF treatments do not seem to affect the level of U-AQP2/P-AVP, which may mostly reflect the ability of the collecting duct. However, there are no data about the changes of U-AQP2/PAVP after the administration of anti-HF treatment such as diuretics. U-AQP2/P-AVP was affected not only immediately but also chronically by the administration of TLV as shown in this study. On the other hand, U-AQP2/P-AVP did not appear to be affected by the chronic anti-HF treatment including conventional diuretics when U-AQP2/P-AVP was measured in the early morning before taking any medicine. However, we do not know if U-AQP2/P-AVP is changed immediately after taking such anti-HF agents. Therefore, U-AQP2/P-AVP should be measured in the early morning before taking any medications including conventional diuretics as well as TLV. 8) We did not measure several urine parameters including beta- 2 microglobulin and N-acetyl-beta-D-glucosaminidase, which are known as indices of tubular dysfunction. Such data would clarify the specific relationship between the collecting duct function and responsiveness to TLV.

Conclusion: TLV can persistently antagonize the V2R and may provide aquaresis over 24 hours in TLV-responders with chronic kidney disease, probably due to the impaired renal excretion of the drug.

\section{ACKNOWLEDGMENTS}

K.K. received a Grant-in-Aid from the Japanese Heart Foundation

\section{Disclosure}

K.K. has a potential conflict of interest with Otsuka Pharmaceutical Co., which consists of lecture fees and endowments from the Department K.K. works at.

\section{REFERENCES}

1. Imamura $\mathrm{T}$, Kinugawa $\mathrm{K}$, Kato $\mathrm{N}$, et al. A case with recovery of response to tolvaptan associated with remission of acute kidney injury and increased urine osmolality. Int Heart J 2013; 54: 115-8.

2. Imamura T, Kinugawa K, Minatsuki S, et al. Urine sodium excretion after tolvaptan administration is dependent upon baseline serum sodium levels: a possible explanation for the improvement of hyponatremia with scarce chance of hypernatremia by a vasopressin receptor antagonist. Int Heart J 2014; 55: 131-7.

3. Imamura T, Kinugawa K, Kato N, et al. Successful conversion from thiazide to tolvaptan in a patient with stage $d$ heart failure and chronic kidney disease before heart transplantation. Int Heart J 2013; 54: 48-50.

4. Imamura T, Kinugawa K, Shiga T, et al. Correction of hyponatremia by tolvaptan before left ventricular assist device implantation. Int Heart J 2012; 53: 391-3.

5. Imamura T, Kinugawa K, Minatsuki S, et al. Tolvaptan can improve clinical course in responders. Int Heart J 2013; 54: 377-81.

6. Gheorghiade M, Gattis WA, O'Connor CM, et al. Effects of tolvaptan, a vasopressin antagonist, in patients hospitalized with worsening heart failure: a randomized controlled trial. JAMA 2004; 291: 1963-71.

7. Gheorghiade M, Konstam MA, Burnett JC Jr, et al. Short-term clinical effects of tolvaptan, an oral vasopressin antagonist, in patients hospitalized for heart failure: the EVEREST Clinical Status Trials. JAMA 2007; 297: 1332-43

8. Inomata T, Izumi T, Matsuzaki M, Hori M, Hirayama A; Tolvaptan Investigators. Phase III clinical pharmacology study of tolvaptan. Cardiovasc Drugs Ther 2011; 25: S57-65.

9. Kinugawa K, Sato N, Inomata T, Shimakawa T, Iwatake N, Mizuguchi K. Efficacy and safety of tolvaptan in heart failure patients with volume overload. Circ J 2014; 78: 844-52.

10. Matsuzaki M, Hori M, Izumi T, Fukunami M. Efficacy and safety of tolvaptan in heart failure patients with volume overload despite the standard treatment with conventional diuretics: a phase III, randomized, double-blind, placebo-controlled study (QUEST study). Cardiovasc Drugs Ther 2011; 25: S33-45.

11. Imamura T, Kinugawa K, Shiga T, et al. Novel criteria of urine osmolality effectively predict response to tolvaptan in decompensated heart failure patients--association between non-responders and chronic kidney disease. Circ J 2013; 77: 397-404.

12. Imamura T, Kinugawa K, Fujino T, et al. Increased urine aquaporin-2 relative to plasma arginine vasopressin is a novel marker of response to tolvaptan in patients with decompensated heart fail- 
ure. Circ J 2014; 78: 2240-9.

13. Imamura T. Aquaporin-2-guided tolvaptan therapy in patients with congestive heart failure accompanied by chronic kidney disease. Int Heart J 2014; 55: 482-3.

14. Martin PY, Abraham WT, Lieming X, et al. Selective V2-receptor vasopressin antagonism decreases urinary aquaporin-2 excretion in patients with chronic heart failure. J Am Soc Nephrol 1999; 10: 2165-70.

15. Kinugawa K. How to treat stage D heart failure? - When to implant left ventricular assist devices in the era of continuous flow pumps?-. Circ J 2011; 75: 2038-45. (Review)

16. Imamura $\mathrm{T}$, Kinugawa $\mathrm{K}$, Hatano $\mathrm{M}$, et al. Low cardiac output stimulates vasopressin release in patients with stage $\mathrm{d}$ heart failure. Circ J 2014; 78: 2259-67.

17. Sasaki S, Ohmoto Y, Mori T, Iwata F, Muraguchi M. Daily variance of urinary excretion of AQP2 determined by sandwich ELISA method. Clin Exp Nephrol 2012; 16: 406-10.

18. Kida K, Shibagaki Y, Tominaga N, et al. Efficacy of tolvaptan added to furosemide in heart failure patients with advanced kidney dysfunction: a pharmacokinetic and pharmacodynamic study. Clin Pharmacokinet 2015; 54: 273-84.

19. Fushimi K, Uchida S, Hara Y, Hirata Y, Marumo F, Sasaki S. Cloning and expression of apical membrane water channel of rat kidney collecting tubule. Nature 1993; 361: 549-52.

20. Kanno K, Sasaki S, Hirata Y, et al. Urinary excretion of aquaporin-2 in patients with diabetes insipidus. N Engl J Med 1995;
332: $1540-5$.

21. DiGiovanni SR, Nielsen S, Christensen EI, Knepper MA. Regulation of collecting duct water channel expression by vasopressin in Brattleboro rat. Proc Natl Acad Sci U S A 1994; 91: 8984-8.

22. Rai T, Sekine K, Kanno K, et al. Urinary excretion of aquaporin-2 water channel protein in human and rat. J Am Soc Nephrol 1997; 8: 1357-62.

23. Elliot S, Goldsmith P, Knepper M, Haughey M, Olson B. Urinary excretion of aquaporin-2 in humans: a potential marker of collecting duct responsiveness to vasopressin. J Am Soc Nephrol 1996; 7 : 403-9.

24. Udelson JE, Orlandi C, Ouyang J, et al. Acute hemodynamic effects of tolvaptan, a vasopressin V2 receptor blocker, in patients with symptomatic heart failure and systolic dysfunction: an international, multicenter, randomized, placebo-controlled trial. J Am Coll Cardiol 2008; 52: 1540-5.

25. Kim SR, Hasunuma T, Sato O, Okada T, Kondo M, Azuma J. Pharmacokinetics, pharmacodynamics and safety of tolvaptan, a novel, oral, selective nonpeptide AVP V2-receptor antagonist: results of single- and multiple-dose studies in healthy Japanese male volunteers. Cardiovasc Drugs Ther 2011; 25: S5-17.

26. Van Wart SA, Shoaf SE, Mallikaarjun S, Mager DE. Population pharmacokinetics of tolvaptan in healthy subjects and patients with hyponatremia secondary to congestive heart failure or hepatic cirrhosis. Biopharm Drug Dispos 2013; 34: 336-47. 